PROCEEDINGS OF THE

AMERICAN MATHEMATICAL SOCIETY

Volume 127, Number 7, Pages 1885-1891

S 0002-9939(99)04579-7

Article electronically published on March 1, 1999

\title{
ON DEHN FUNCTIONS OF FREE PRODUCTS OF GROUPS
}

\author{
V. S. GUBA AND M. V. SAPIR
}

(Communicated by Ronald M. Solomon)

\begin{abstract}
In this article we show that the Dehn function of a nontrivial free product of groups is equivalent to its subnegative closure.
\end{abstract}

Let $\mathcal{P}=\langle\Sigma \mid \mathcal{R}\rangle$ be a finite presentation of a group $G$. Suppose that a word $w$ over $\Sigma$ is equal to 1 in $G$. By van Kampen's Lemma [6], there exists a diagram over $\mathcal{P}$ such that $w$ is a boundary label of this diagram. By $k(w)$ we denote the smallest number of cells in such a diagram. In other words, $k(w)$ is the smallest number with the following property: $w$ is equal in the free group over $\Sigma$ to a product of $k(w)$ conjugates of the defining relators from $\mathcal{R}$ or their inverses.

A function $f: \mathbf{N} \rightarrow \mathbf{N}$ is called the Dehn function of the presentation $\mathcal{P}$ whenever $f(n)=\max k(w)$, where maximum is taken over all words such that $w$ equals 1 in $G$ and $|w| \leq n$.

Let $f, g$ be functions from $\mathbf{N}$ to $\mathbf{N}$. By definition, $f \preceq g$ means that there exists a positive integer $C$ such that $f(n) \leq C g(C n)+C n$ for all $n$. This relation induces an equivalence relation on the set of functions from $\mathbf{N}$ into itself: $f \simeq g$ if and only if $f \preceq g$ and $g \preceq f$.

It is well known (see [7], [1], [4]) that if $G$ is a finitely presented group and $f, g$ are the Dehn functions of two finite presentations of $G$, then $f \simeq g$. In this paper, we shall not distinguish between equivalent functions. Thus we will speak about the Dehn function of a finitely presented group $G$.

Following Brick [3], we say that a function $f: \mathbf{N} \rightarrow \mathbf{N}$ is subnegative whenever $f(m)+f(n) \leq f(m+n)$ for all $m, n \in \mathbf{N}$. For every function $f: \mathbf{N} \rightarrow \mathbf{N}$ one can define a function $\bar{f}(n)$ by the following formula:

$$
\bar{f}(n)=\max \left(f\left(n_{1}\right)+f\left(n_{2}\right)+\cdots f\left(n_{r}\right)\right),
$$

where maximum is taken for all $r \geq 1$, and $n_{1}, \ldots, n_{r} \in \mathbf{N}$ such that $n_{1}+\cdots+n_{r}=$ $n$. It is easy to see that $\bar{f}(n)$ is the smallest subnegative function which is greater than or equal to $f(n)$. The function $\bar{f}$ is said to be the subnegative closure of $f$.

The subnegative property plays an important role in [2], where it is proved that the class of Dehn functions is very large. Roughly speaking, every relatively fast computable subnegative function $\geq n^{4}$ is the Dehn function of a finitely presented group. More precisely, if $T(n)$ is a subnegative time function of a nondeterministic

Received by the editors June 27, 1997.

1991 Mathematics Subject Classification. Primary 20F32; Secondary 57M07.

The research of the first author was supported in part by grants from the ISF and the Russian Foundation for Fundamental Researches, grant no. 96-01-00420. The research of the second author was supported in part by NSF grants. 
Turing machine such that $\sqrt[4]{T(n)}$ is equivalent to a time function, then $T(n)$ is the Dehn function of a finitely presented group. On the other hand, every Dehn function of a finitely presented group is equivalent to the time function of a nondeterministic Turing machine. Thus if every Dehn function were equivalent to a subnegative function and in addition the fourth root of every time function were equivalent to a time function (which is quite possible), then the main result from [2] would give a complete description of all Dehn functions of groups which are greater than $n^{4}$.

It is easy to see that if $f(n)$ is the Dehn function of a finite presentation, then $f$ may not be subnegative. For example, for the presentation $\left\langle a \mid a^{2}=1, a^{3}=1\right\rangle$ one has $f(1)=2, f(2)=2$. But it is natural to ask whether every Dehn function is equivalent to a subnegative function. The second author conjectured that this is always true.

Conjecture 1. The Dehn function of any finite presentation is equivalent to a subnegative function.

Recall that not every increasing function is equivalent to a subnegative one (see [3]).

Let us note that if $f \preceq g$, then $\bar{f} \preceq \bar{g}$. Therefore $f \simeq g$ implies $\bar{f} \simeq \bar{g}$. We obtain the following equivalent

Conjecture $\mathbf{1}^{\prime}$. Every Dehn function of a finite presentation is equivalent to its subnegative closure.

The aim of this paper is to show that the conjecture is true for groups which are nontrivially decomposable into a free product.

Theorem. Let $G_{1}, G_{2}$ be nontrivial finitely presented groups. Then the Dehn function of their free product $G_{1} * G_{2}$ is equivalent to its subnegative closure.

Corollary. Let $G_{1}, G_{2}$ be nontrivial finitely presented groups and let $f_{1}, f_{2}$ be their Dehn functions. The Dehn function $f$ of the group $G_{1} * G_{2}$ satisfies the following equality: $f \simeq \max \left(\bar{f}_{1}, \bar{f}_{2}\right)$.

This corollary improves Proposition 3.1 in Brick [3] which says that

$$
\max \left(f_{1}, f_{2}\right) \preceq f \preceq \max \left(\bar{f}_{1}, \bar{f}_{2}\right) .
$$

The corollary follows immediately from this inequality and from our main result.

This corollary immediately implies that our conjecture is equivalent to the following statement:

Conjecture $\mathbf{1}^{\prime \prime}$. For any finitely presented group $G$, the Dehn functions of $G$ and $G * \mathbf{Z}$ are equivalent.

One might say that if this conjecture is wrong, then something is wrong with the definition of Dehn functions. More seriously, it could be better to call the "true Dehn function" of a group the subnegative closure of the Dehn function. Our Corollary shows that "true Dehn functions" behave nicely with respect to free products. Since all known Dehn functions are subnegative, this change of definition will not affect any known results about Dehn functions.

To prove our theorem, we use diagrams over group presentations (see [6]). Let us recall that $\varphi(e)$ denotes the label of an edge $e$ of a diagram. All contours of diagrams are read clockwise, all contours of cells are read counterclockwise. By $\iota(e), \tau(e)$ we denote the initial and the terminal vertices of an edge $e$ respectively. 


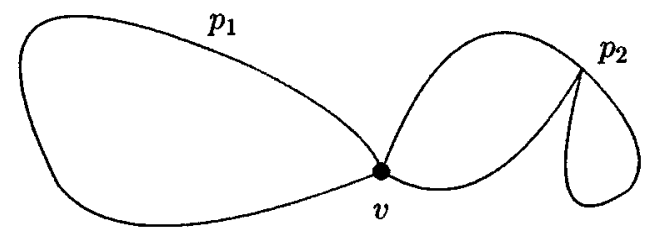

FiguRE 1

We need the following auxiliary definition.

Let $\Delta$ be a diagram over a group presentation and let $v$ be a vertex in the boundary of $\Delta$. We say that $v$ is a double vertex of $\Delta$ if $\Delta$ has a contour of the form $p_{1} p_{2}$ where $p_{1}, p_{2}$ are nontrivial loops at $v$ (see Figure 1 ).

Let $\left\langle\Sigma_{i} \mid \mathcal{R}_{i}\right\rangle$ be a finite presentation of a group $G_{i}(i=1,2)$. We assume that $\Sigma_{1}$ and $\Sigma_{2}$ do not intersect. It is obvious that $\langle\Sigma \mid \mathcal{R}\rangle$ is a finite presentation for $G_{1} * G_{2}$, where $\Sigma=\Sigma_{1} \cup \Sigma_{2}, \mathcal{R}=\mathcal{R}_{1} \cup \mathcal{R}_{2}$. We keep these definitions throughout the paper.

Lemma 1. Let $\Delta$ be a diagram over $\langle\Sigma \mid \mathcal{R}\rangle$. Suppose that it has a contour of the form $e_{1} p e_{2}$, where $e_{1}, e_{2}$ are edges. If $\varphi\left(e_{i}\right) \in \Sigma_{i}^{ \pm 1}$ for each $i \in\{1,2\}$, then $v=\iota\left(e_{1}\right)=\tau\left(e_{2}\right)$ is a double vertex of $\Delta$.

Proof. Let $h_{0}, h_{1}, \ldots, h_{r}(r \geq 1)$, where $h_{0}=e_{1}, h_{1}=e_{2}^{-1}$, be the sequence of all edges going out from $v$ in the clockwise order. (Note that this sequence may have occurrences of the form $h, h^{-1}$ if $h$ forms a loop.) Each of the edges $h_{j}(0 \leq j \leq r)$ has a label either from $\Sigma_{1}^{ \pm 1}$ or from $\Sigma_{2}^{ \pm 1}$. Since $h_{0}$ and $h_{r}$ have labels from different alphabets, there exist two neighbour edges $h_{j}$ and $h_{j+1}(0 \leq j<r)$ such that their labels belong to different alphabets.

Since there are no defining relations from $\mathcal{R}$ that contain letters from both of the two alphabets, there are no cells "between" $h_{j}$ and $h_{j+1}$ (i.e. no contours of cells of $\Delta$ have subpaths $\left.h_{j}^{-1} h_{j+1}\right)$. It is clear that the edge $h_{j}^{-1}$ cannot belong to a boundary contour of any cell since otherwise there are edges between $h_{j}$ and $h_{j+1}$. The same argument applies for the edge $h_{j+1}$. This implies that $h_{j}^{-1}, h_{j+1}$ belong to a boundary contour of $\Delta$ and moreover, they are consecutive edges of the cyclic contour. We obtain that the contour of $\Delta$ at $v$ has a form $p_{1} p_{2}$ where $p_{1}=e_{1} \ldots h_{j}^{-1}, p_{2}=h_{j+1} \ldots e_{2}$ are nontrivial loops at $v$. Thus $v$ is a double vertex. The proof is complete.

Lemma 2. Let $\Delta$ be a diagram over $\langle\Sigma \mid \mathcal{R}\rangle$. Suppose that $G_{1}$ and $G_{2}$ are nontrivial groups. Let $a_{i}$ denote an element in $\Sigma_{i}$ such that $a_{i} \neq 1$ in $G_{i}(i=1,2)$. Then there exists a vertex $v$ in the boundary of $\Delta$ and there exists an index $j \in$ $\{1,2\}$ such that for any decomposition $p q$ of the contour of $\Delta$ as a loop at $v$, we have $\varphi(p) \neq a_{j}$ in $G$.

Proof. Suppose that the conclusion is not true. Let $v_{0}$ be an arbitrary vertex in the boundary of $\Delta$. Let us define an infinite sequence $v_{m}$ of vertices in the boundary of $\Delta$ and an infinite sequence $p_{m}$ of subpaths of the cyclic contour of $\Delta$ by induction on $m$. Let $m \geq 1$ and let $v_{j}, p_{j}$ be defined for all $0 \leq j<m$. By our assumption, for any $i=1,2$ there exists a decomposition $p(i) q(i)$ of the contour of $\Delta$ at $v_{m-1}$ such that $\varphi(p(i))=a_{i}$ in $G$. Let $p_{m}$ be $p(1)$ for odd $m$ and $p(2)$ for even $m$. By 
definition, $v_{m}$ is the terminal vertex of $p_{m}$. Note that all $p_{m}$ are nontrivial since $a_{1}, a_{2}$ are not equal to 1 in $G$.

Since the set of the boundary vertices of $\Delta$ is finite, we have $v_{m}=v_{m+s}$ for some $m \geq 0, s \geq 1$. Thus the path $p=p_{m+1} \ldots p_{m+s}$ is a nontrivial power of a boundary label of $\Delta$. Since the boundary label of any diagram is equal to 1 in the corresponding group, this implies that $\varphi(p)=1$ in $G$. But $\varphi(p)$ is a nontrivial product of letters $a_{1}, a_{2}$ such that all neighbour letters are different. This shows that $\varphi(p)$ represents a normal form of the free product $G_{1} * G_{2}$. But a nonempty normal form represents a nontrivial element of a free product (see [6]). We have a contradiction. This completes the proof.

Now we are able to prove our Theorem.

Let $f$ be the Dehn function of $\langle\Sigma \mid \mathcal{R}\rangle$ where $G_{1}, G_{2}$ are nontrivial. We shall prove the inequality

$$
f\left(n_{1}\right)+f\left(n_{2}\right) \leq f\left(n_{1}+n_{2}+6\right)
$$

for all $n_{1}, n_{2}$. Let us show that inequality (2) implies the statement of the Theorem. Notice that the Dehn function of any presentation is increasing. It follows easily from this fact and inequality (2) that $f\left(n_{1}\right)+\cdots+f\left(n_{r}\right) \leq f\left(n_{1}+\cdots+n_{r}+6(r-1)\right)$. Now if any integer $n$ is presented as a sum of positive integers $n_{1}, \ldots, n_{r}$, then we have $r \leq n$ and $f\left(n_{1}\right)+\cdots+f\left(n_{r}\right) \leq f(n+6(r-1)) \leq f(7 n)$. Using (1) we obtain $\bar{f}(n) \leq f(7 n)$ for all $n$. This means that $\bar{f} \preceq f$. Since $f(n) \leq \bar{f}(n)$ for all $n$, we always have $f \preceq \bar{f}$. Now it is clear that $f \simeq \bar{f}$. So it remains to prove inequality (2).

By definition of $f$, there exist diagrams $\Delta_{1}, \Delta_{2}$ over $\langle\Sigma \mid \mathcal{R}\rangle$ with boundary labels $W_{i}$ that have $f\left(n_{i}\right)$ cells and the length of $W_{i}$ does not exceed $n_{i}(i=1,2)$. Moreover, no diagram with boundary label $W_{i}$ can have fewer than $f\left(n_{i}\right)$ cells $(i=1,2)$.

Starting with $\Delta_{1}, \Delta_{2}$, we shall construct a word $W$ and a diagram $\Delta$ with boundary label $W$ and the following properties:

- The perimeter of $\Delta$ does not exceed $n_{1}+n_{2}+6$.

- $\Delta$ has exactly $f\left(n_{1}\right)+f\left(n_{2}\right)$ cells.

- No diagram with the same boundary label $W$ can have fewer cells.

Inequality (2) follows from these properties by definition of Dehn functions. So it remains to construct such a diagram $\Delta$.

First of all, we apply Lemma 2 to $\Delta_{1}$. We find a boundary vertex $v_{1}$ of $\Delta_{1}$ and an integer $i_{1}=1,2$ such that no initial subpath of the boundary contour of $\Delta_{1}$ at $v_{1}$ has a label equal to $a_{i_{1}}$ in $G$. After that, applying Lemma 2 to the mirror copy of $\Delta_{2}$, we find a boundary vertex $v_{2}$ of $\Delta_{2}$ and an integer $i_{2}=1,2$ such that no terminal subpath of the boundary contour of $\Delta_{2}$ at $v_{2}$ has a label equal to $a_{i_{2}}^{-1}$.

Denote by $t_{i}$ the boundary contour of $\Delta_{i}$ at $v_{i}(i=1,2)$. We distinguish two cases: $i_{1} \neq i_{2}$ and $i_{1}=i_{2}$.

Case 1. For simplicity, let us assume that $i_{1}=1, i_{2}=2$. Let us construct a diagram $\Delta$ taking $\Delta_{1}, \Delta_{2}$ and joining them by a "bridge" of two edges $e_{1}, e_{2}$ (see Figure 2).

Here $\varphi\left(e_{i}\right)=a_{i}$ for $i=1,2$ and $\Delta$ has a contour $e_{1}^{-1} t_{1} e_{1} e_{2}^{-1} t_{2} e_{2}$ at $w=\tau\left(e_{1}\right)=$ $\tau\left(e_{2}\right)$. It is clear from our construction that $\Delta$ has exactly $f\left(n_{1}\right)+f\left(n_{2}\right)$ cells, and its perimeter does not exceed $n_{1}+n_{2}+4$. We shall prove that no diagram with the same boundary label has fewer cells. 


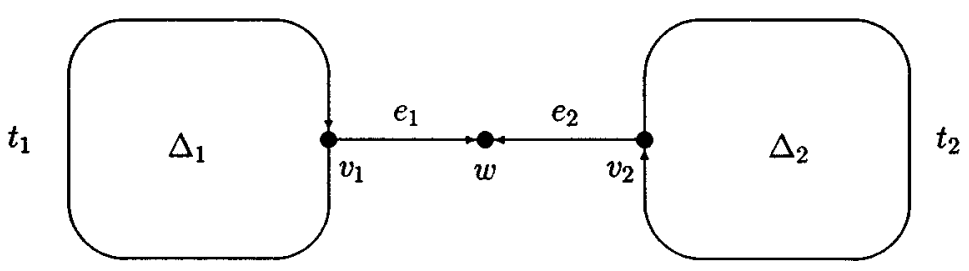

FIGURE 2

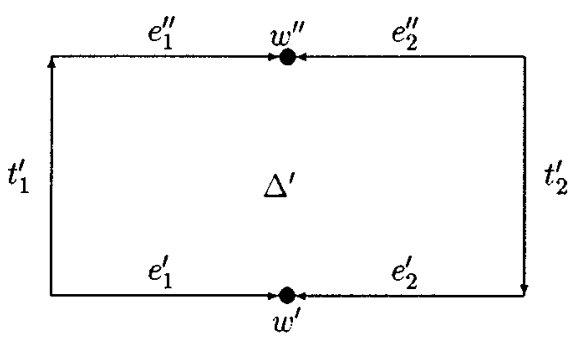

FiguRE 3

Suppose that a diagram $\Delta^{\prime}$ has the same boundary label as $\Delta$. Thus its contour can be represented as $\left(e_{1}^{\prime}\right)^{-1} t_{1}^{\prime} e_{1}^{\prime \prime}\left(e_{2}^{\prime \prime}\right)^{-1} t_{2}^{\prime} e_{2}^{\prime}$ where $W_{i}=\varphi\left(t_{i}\right)=\varphi\left(t_{i}^{\prime}\right), \varphi\left(e_{i}\right)=$ $\varphi\left(e_{i}^{\prime}\right)=\varphi\left(e_{i}^{\prime \prime}\right)=a_{i}(i=1,2)$. (See Figure 3.)

Let $w^{\prime}=\tau\left(e_{1}^{\prime}\right)=\tau\left(e_{2}^{\prime}\right), w^{\prime \prime}=\tau\left(e_{1}^{\prime \prime}\right)=\tau\left(e_{2}^{\prime \prime}\right)$. Applying Lemma 1 we conclude that $w^{\prime}$ is a double vertex of $\Delta^{\prime}$. Thus $w^{\prime}$ coincides with some other boundary vertex.

Suppose that $w^{\prime}=w^{\prime \prime}$. In this case we can consider diagrams $\Delta_{1}^{\prime}$ and $\Delta_{2}^{\prime}$ with contours $\left(e_{1}^{\prime}\right)^{-1} t_{1}^{\prime} e_{1}^{\prime \prime}$ and $\left(e_{2}^{\prime \prime}\right)^{-1} t_{2}^{\prime} e_{2}^{\prime}$ respectively. In each of these two diagrams we can glue edges $e_{i}^{\prime}$ and $e_{i}^{\prime \prime}$ (they have the same label) obtaining two diagrams $\Delta_{i}^{\prime \prime}(i=1,2)$. Notice that when gluing these edges we may need to cut off some spherical subdiagrams. As a result, we get two diagrams that have boundary labels $W_{1}$ and $W_{2}$. By the remark above, $\Delta_{i}^{\prime \prime}$ has at least $f\left(n_{i}\right)$ cells $(i=1,2)$. Therefore, $\Delta^{\prime}$ has at least $f\left(n_{1}\right)+f\left(n_{2}\right)$ cells as desired.

Now suppose that $w^{\prime} \neq w^{\prime \prime}$. Thus $w^{\prime}$ coincides with one of the vertices that belongs to either $t_{1}^{\prime}$ or $t_{2}^{\prime}$. In the first case we can find a subdiagram in $\Delta^{\prime}$ with contour $e_{1}^{\prime} t_{1}^{\prime \prime}$ where $t_{1}^{\prime \prime}$ is an initial subpath of $t_{1}^{\prime}$. Thus $\varphi\left(t_{1}^{\prime \prime}\right)=\varphi\left(e_{1}^{\prime}\right)=a_{1}$ in $G$. This is a contradiction since $t_{1}$ has an initial subpath whose label is equal to $a_{1}$ in $G$. In the second case we have a subdiagram in $\Delta^{\prime}$ with boundary contour $t_{2}^{\prime \prime} e_{2}^{\prime}$ where $t_{2}^{\prime \prime}$ is a terminal subpath of $t_{2}^{\prime}$. This implies that $\varphi\left(t_{2}^{\prime \prime}\right)=\varphi\left(e_{2}^{\prime}\right)^{-1}=a_{2}^{-1}$ in $G$. We have also a contradiction since $t_{2}$ cannot have a terminal subpath whose label is equal to $a_{2}^{-1}$ in $G$.

Case 2. Now without loss of generality assume that $i_{1}=i_{2}=1$. We also construct a diagram $\Delta$ taking $\Delta_{1}, \Delta_{2}$ and joining them by a "bridge" of three edges $e_{1}, e, e_{2}$ (see Figure 4).

Here $\varphi\left(e_{1}\right)=\varphi\left(e_{2}\right)=a_{1}, \varphi(e)=a_{2}$ and $\Delta$ has a contour $e_{1}^{-1} t_{1} e_{1} e e_{2}^{-1} t_{2} e_{2} e^{-1}$ at $w=\tau\left(e_{1}\right)=\iota(e)$. It is clear from our construction that $\Delta$ has exactly $f\left(n_{1}\right)+f\left(n_{2}\right)$ cells, and its perimeter does not exceed $n_{1}+n_{2}+6$. We shall prove that no diagram with the same boundary label can have fewer cells. 


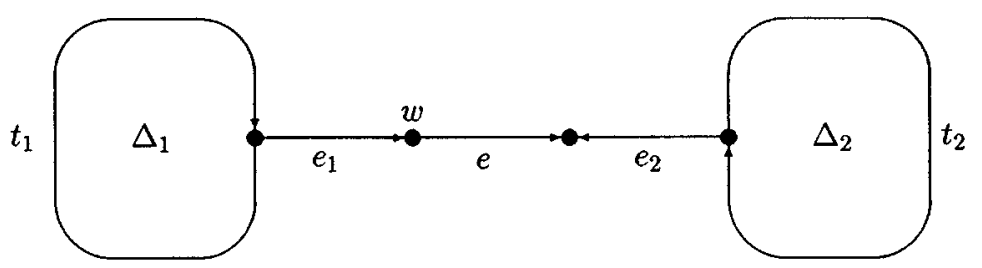

FigURE 4

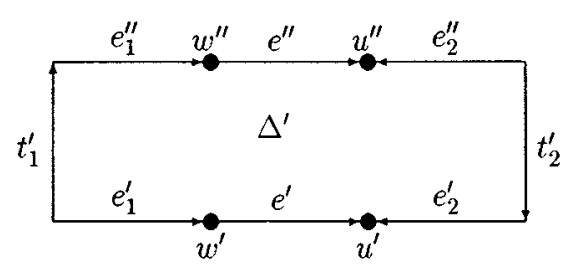

FiguRE 5

Suppose that a diagram $\Delta^{\prime}$ has the same boundary label. Thus its contour can be represented as $\left(e_{1}^{\prime}\right)^{-1} t_{1}^{\prime} e_{1}^{\prime \prime} e^{\prime \prime}\left(e_{2}^{\prime \prime}\right)^{-1} t_{2}^{\prime} e_{2}^{\prime}\left(e^{\prime}\right)^{-1}$ where $W_{i}=\varphi\left(t_{i}\right)=\varphi\left(t_{i}^{\prime}\right)$, $\varphi\left(e_{i}\right)=\varphi\left(e_{i}^{\prime}\right)=\varphi\left(e_{i}^{\prime \prime}\right)=a_{1}(i=1,2), \varphi(e)=\varphi\left(e^{\prime}\right)=\varphi\left(e^{\prime \prime}\right)=a_{2}$. (See Figure 5.)

Let $w^{\prime}=\tau\left(e_{1}^{\prime}\right)=\iota\left(e^{\prime}\right), w^{\prime \prime}=\tau\left(e_{1}^{\prime \prime}\right)=\iota\left(e^{\prime \prime}\right), u^{\prime}=\tau\left(e^{\prime}\right)=\tau\left(e_{2}^{\prime}\right), u^{\prime \prime}=\tau\left(e^{\prime \prime}\right)=$ $\tau\left(e_{2}^{\prime \prime}\right)$. Applying Lemma 1 we conclude that $w^{\prime}$ is a double vertex of $\Delta^{\prime}$. Thus $w^{\prime}$ coincides with some other boundary vertex.

Suppose that $w^{\prime}=w^{\prime \prime}$. In this case we have two subdiagrams in $\Delta^{\prime}$ with contours $\left(e_{1}^{\prime}\right)^{-1} t_{1}^{\prime} e_{1}^{\prime \prime}$ and $e^{\prime \prime}\left(e_{2}^{\prime \prime}\right)^{-1} t_{2}^{\prime} e_{2}^{\prime}\left(e^{\prime}\right)^{-1}$. Gluing $e_{1}^{\prime}$ and $e_{1}^{\prime \prime}, e^{\prime}$ and $e^{\prime \prime}$, and $e_{2}^{\prime}$ and $e_{2}^{\prime \prime}$ in these subdiagrams, and cutting off spherical subdiagrams if necessary, we obtain diagrams $\Delta_{i}^{\prime \prime}$ with boundary labels $W_{i}(i=1,2)$. These diagrams have at least $f\left(n_{1}\right), f\left(n_{2}\right)$ cells respectively, so $\Delta^{\prime}$ has at least $f\left(n_{1}\right)+f\left(n_{2}\right)$ cells, as desired.

If $w^{\prime}$ coincides with a vertex in $t_{1}^{\prime}$, we finish the proof in a similar way as in Case 1. Note that $w^{\prime}$ does not coincide with $u^{\prime}$ since otherwise $a_{2}=\varphi\left(e^{\prime}\right)=1$ in $G$. Therefore $w^{\prime}$ coincides with a vertex in $\left(e_{2}^{\prime \prime}\right)^{-1} t_{2}^{\prime}$. By Lemma $1, u^{\prime}$ is also a double vertex. If $w^{\prime}$ coincides with a vertex in $t_{2}^{\prime}$, then $u^{\prime}$ also coincides with a vertex in $t_{2}^{\prime}$. If $w^{\prime}=u^{\prime \prime}$, then $u^{\prime}$ cannot coincide with $u^{\prime \prime}$ so it coincides with a vertex in $t_{2}^{\prime}$ anyway. Now we finish the proof similarly to Case 1.

The Theorem is proved.

\section{REFERENCES}

1. G. Baumslag, C. F. Miller III, and H. Short. Isoperimetric inequalities and the homology of groups. Invent. Math., 113:531-560, 1993. MR 95b:57004

2. M.V. Sapir, J.-C. Birget, E.Rips. Dehn functions of groups, to appear.

3. S. G. Brick, Dehn functions and products of groups. Trans. Amer. Math. Soc., 335 (1993),369384. MR 93c: 57003

4. S. Gersten. Isoperimetric and isodiametric functions of finite presentations. In Geometric group theory, Vol. 1 (Sussex, 1991), London Math. Soc. Lecture Note Ser., 181 (1993), 79-96. MR 94f: 20066

5. M. Gromov. Hyperbolic groups. In Essays in Group Theory (S. Gersten, ed.), MSRI Publ. 8. Springer-Verlag, 1987, 75-263. MR 89e:20070 
6. R. C. Lyndon and P. E. Schupp. Combinatorial group theory. Springer-Verlag, 1977. MR $\mathbf{5 8 : 2 8 1 8 2}$

7. K. Madlener, F. Otto. Pseudo-natural algorithms for the word problem for finitely presented monoids and groups. J. Symbolic Computation, 1:383-418, 1985. MR 87h:03067

Department of Mathematics, Vologda State Pedagogical University, S. Orlov St., 6, Vologda 160600, Russia

E-mail address: guba@vgpi.vologda.su

Department of Mathematics, Vanderbilt University, Nashville, Tennessee 37240

E-mail address: msapir@math.vanderbilt.edu 